\title{
Bubble size distribution resulting from the breakup of an air cavity injected into a turbulent water jet
}

\author{
Carlos Martínez-Bazán, José L. Montañés, and Juan C. Lasheras \\ Department of Mechanical and Aerospace Engineering, University of California, San Diego, \\ 9500 Gilman Drive, La Jolla, California 92093
}

\begin{abstract}
We investigated experimentally the shape of the final size $\operatorname{PDF}(D)$ resulting from the breakup of an air bubble injected into the fully developed region of a high Reynolds number turbulent water jet. It is shown that the $\operatorname{PDF}(\hat{D})$ of the normalized bubble size $\hat{D}=D / D_{32}$, where $D_{32}$ is the Sauter mean diameter of the distribution, has a universal single shape independent of the value of the turbulent kinetic energy of the water jet at the bubble injection point and of the air void fraction, $\alpha$. The shape of the exponential tails characterizing each $\operatorname{PDF}(D)$ is shown to be only a function of the initial bubble size $D_{0}$ and the critical bubble size $D_{c}$, defined as $D_{c}=(1.46 \sigma / \rho)^{3 / 5} \epsilon^{-2 / 5}$, where $\epsilon$ is the value of the dissipation rate of turbulent kinetic energy per unit mass at the air injection point.
\end{abstract}

The droplet size distribution function resulting from turbulent breakup of a lump of an immiscible fluid injected into a turbulent flow is of fundamental importance to the design of a wide range of chemical reactors, combustors, and many other engineering processes, and it has been largely studied since the initial work by Kolmogorov ${ }^{1}$ and Hinze. ${ }^{2}$ Although several approaches have been proposed to obtain the size PDF (Probability density function), Cohen, ${ }^{3}$ LonguetHiggins, ${ }^{4}$ Brown and Wohletz, ${ }^{5}$ and Novikov and Dommermuth $^{6}$ among others, no universal shape has been shown to exist independent of the value of the turbulent kinetic energy of the underlying turbulence.

The objective of this work is to obtain detailed experimental measurements of the probability density function of the droplet sizes resulting from the breakup of an immiscible fluid injected into a prototypical turbulent flow of known characteristics. In order to isolate the problem, and to prevent the additional complexity introduced by the use of solid surfaces or any other moving surfaces, to generate the turbulence we selected to study the turbulent break-up by injecting air bubbles into the fully developed turbulent region along the central axis of a high Reynolds number water jet (for details of the experiments see Martínez-Bazán $\left.{ }^{7,8}\right)$. In these experiments, a continuous jet of air was injected coaxially at the centerline of a water jet with a velocity equal to the mean velocity of the water measured at the air injection point. Using image processing and phase doppler techniques, the final bubble size PDF achieved once the breakup process is concluded was measured over a wide range of air void fraction, $\alpha$, and values of the turbulent kinetic energy, $\epsilon$, at the injection point.

To study the dependence of the shape of the bubble size
PDF on the intensity of the underlying turbulence, we conducted a systematic set of experiments where $\epsilon$ was varied while keeping the air injection needle's diameter $\left(D_{n}\right.$ $=0.39 \mathrm{~mm}$ ) and the flow rate of air, $Q_{a}$, injected into the flow constant. In each case the water jet Reynolds number was increased from 28000 to 93000 and three different values of the flow rate of air $\left(Q_{a}=3.6 \mathrm{ml} / \mathrm{min}, Q_{a}\right.$ $=7.25 \mathrm{ml} / \mathrm{min}$, and $Q_{a}=34.35 \mathrm{ml} / \mathrm{min}$ ) were studied.

The final bubble size PDFs obtained by fixing the injection flow rate of the air while systematically increasing the Reynolds number of the water jet are shown in Fig. 1. Upon injection into the turbulent water jet, the air jet is broken by the turbulent stresses exerted by the underlying turbulence until an unchanged or final size PDF is obtained. In Fig. 1 each PDF corresponds to a given value of the water jet Reynolds number, which in turn represents a certain value of $\epsilon$ at the injection point. For convenience, since the diameter of the water nozzle, $D_{J}$, and the air injection point, $X / D_{J}$ $=10$, were kept constant, the Reynolds number of the water jet, $R_{e}=U_{0} D_{J} / \nu$, was used as the only parameter to indicate increasing levels of turbulent kinetic energy existing at the air injection point. It is evident from these measurements that the shape of the bubble size PDF strongly depends on the turbulent kinetic energy (or dissipation rate $\epsilon$ ) of the underlying turbulence. This is not surprising since, as the value of $\epsilon$ at the air injection point increases, the jet of air continuously injected into the turbulent water jet is subjected to increasing values of turbulent stresses acting on its surface. Consequently, the value of the critical bubble's diameter, defined by Kolmogorov ${ }^{1}$ as $D_{c} \propto(\sigma / \rho)^{3 / 5} \epsilon^{-2 / 5}$ where $\sigma$ is the interfacial surface tension, which is a measure of the diameter of the largest bubble stable in a given turbulent flow, decreases. This is apparent in Fig. 1 where one can clearly see that as the Reynolds number is increased the PDF becomes narrower, and their peaks are shifted to the smaller sizes as $\epsilon$ increases.

The experimental measurements corresponding to the 


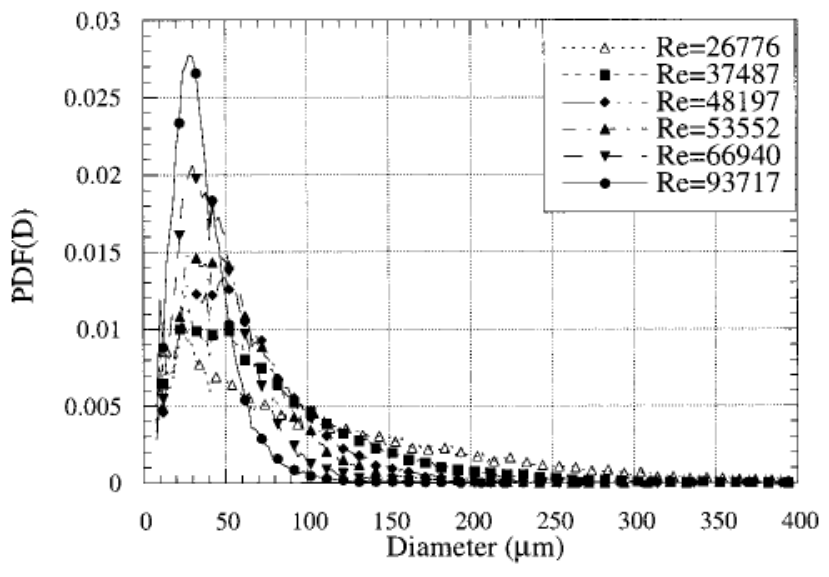

FIG. 1. Final bubble size PDF for various Reynolds numbers of the submerged water jet. $Q_{a}=7.25 \mathrm{ml} / \mathrm{min}$.

lowest flow rate of air $\left(Q_{a}=3.6 \mathrm{ml} / \mathrm{min}\right)$ are presented in Fig. 2, where for clarity, we have plotted the final bubble size PDF for only three values of the $R_{e}$. The slope of the exponential tails of the PDFs is shown to increase with the $R_{e}$, a consequence of the decrease of $D_{c}$ with $\epsilon$. In order to represent the bubble size PDF in nondimensional variables, we chose $D_{32}=\Sigma N_{i} D_{i}^{3} / \Sigma N_{i} D_{i}^{2}$ as the normalization bubble's diameter, and we defined $\hat{D}=D / D_{32}$. The probability density function of $\hat{D}, \operatorname{PDF}(\hat{D})$, for the set of conditions shown in Fig. 2 are given in Fig. 3. Note that all the $\operatorname{PDFs}(\hat{D})$ collapse onto a single curve, showing that the slope of the tails of the bubble's size distribution are only a function of $D_{32}$, which was measured to be in all cases linearly proportional to the critical bubble's diameter ${ }^{9} D_{c}$. The same type of behavior, although not shown here, was also observed for the other cases tested at $Q_{a}=7.25 \mathrm{ml} / \mathrm{min}$, and $Q_{a}=34.35 \mathrm{ml} / \mathrm{min}$.

To study the effect of the initial air void fraction on the shape of the final bubble PDF we conducted an additional set of experiments where all the other parameters were kept constant while $\alpha$ was varied. Here, we defined $\alpha=Q_{a} / Q_{w}$, where $Q_{a}$ is the flow rate of air and $Q_{w}$ is the flow rate of water. For this purpose, we fixed the Reynolds number at a

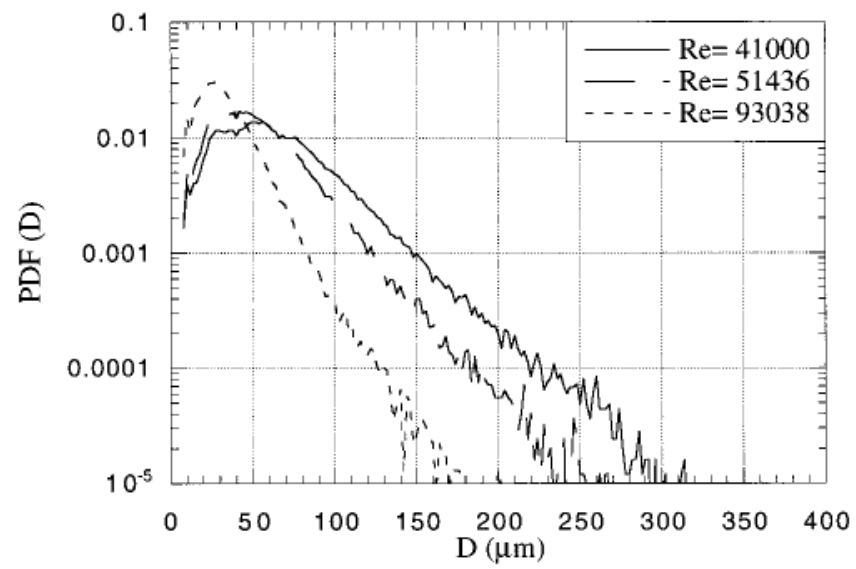

FIG. 2. Evolution of the bubble size PDF with the Reynolds number. $Q_{a}$ $=3.6 \mathrm{ml} / \mathrm{min}$.

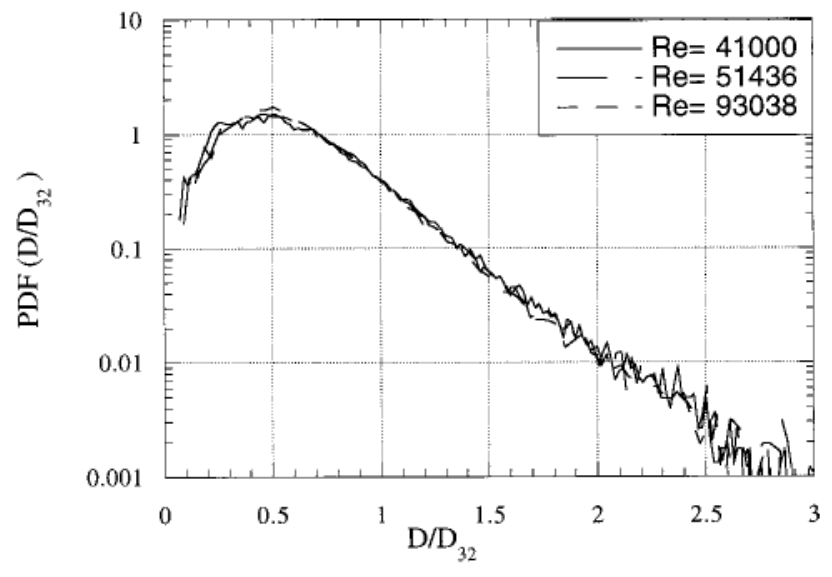

FIG. 3. Bubble size PDF of $\hat{D}=D / D_{32} \cdot Q_{a}=3.6 \mathrm{ml} / \mathrm{min}$.

representative intermediate value of 54036 , and we varied the void fraction by nearly two orders of magnitude, from $3.5 \times 10^{-4}$ to $7.45 \times 10^{-3}$, by increasing the air flow rate. The resulting final PDFs are shown in Fig. 4. Qualitatively, one observes that increasing the void fraction appears to have a similar effect as decreasing the value of the dissipation rate, $\epsilon$, i.e., the slope of the tails of the PDFs increases by decreasing $\alpha$. As was the case with the evolution of PDF with $\epsilon$ described above, the normalized $\operatorname{PDFs}(\hat{D})$ of this set of conditions also collapse onto a single, self-similar curve indicating that $D_{32}$ is the only parameter needed to describe the final state of the bubble's size PDF (see Fig. 5). Analyzing Figs. 3 and 5 we can observe that the tails of the normalized PDFs decay exponentially as $\operatorname{PDF}\left(D / D_{32}\right) \propto \exp$ $\left(-3.3 D / D_{32}\right)$. From the experimental results presented here it can be inferred that the coefficient of proportionality between $D_{32}$ and $D_{c}$ should be a function of $\alpha, D_{32}$ $=f(\alpha) D_{c}$. Consequently, it means that the Kolmogorov's scaling could be used with a modified or effective dissipation rate, $\epsilon_{\alpha}=\epsilon f^{-5 / 2}(\alpha)$.

The possible causes of the observed dependence of the PDFs on $\alpha$ are: (a) coalescence effects; (b) modifications of the turbulent energy spectra due to the presence of the air bubbles, which translate into lower values of the $\epsilon$, increas-

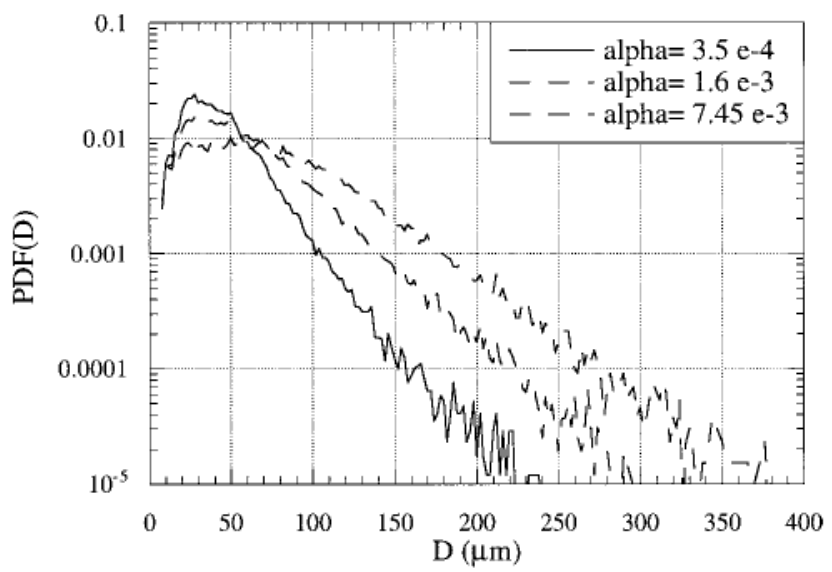

FIG. 4. Evolution of the bubbles size PDF with the air void fraction, $\alpha . R_{e}=54036$. 


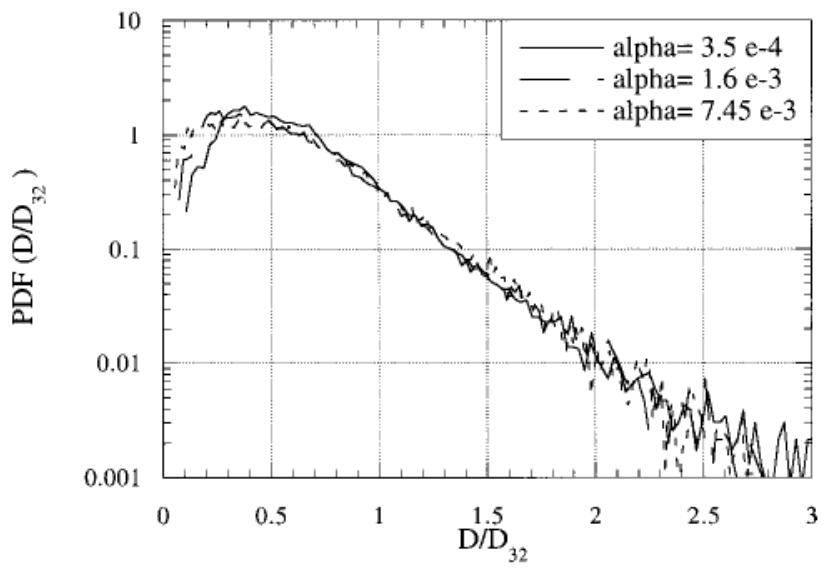

FIG. 5. Bubble size PDF of $\hat{D}=D / D_{32} . R_{e}=54036$.

ing therefore the value of the critical diameter, $D_{c}$, and consequently the value of $D_{32}$; or (c) the effect of the initial bubble size on the bubble breakup frequency and daughter bubble size distribution. The role of coalescence appears to be negligibly small in all our experiments since in all cases the value of $\epsilon$ was very large and the process was fully controlled by breakup, see Lasheras et al. ${ }^{10}$ To account for the possible effect of $\alpha$ on $\epsilon$, we measured the energy spectrum of the water jet turbulence at the air injection point by placing a hot-film probe at the air injection point, but radially displaced a small distance from the jet's centerline, and far enough to avoid any contact of the bubbles with the probe. If the energy spectrum was affected by the presence of the bubbles, one would measure its effects by observing changes in the energy spectrum of the turbulence. Since no changes were found on the measured spectra, it seems reasonable to conclude that any local changes in the energy spectra due to the presence of the bubbles in the flow cannot account for the large changes measured in the final PFDs shown in Fig. 4.

In order to analyze the effect of the initial bubble size $D_{0}$ on the shape of the PDF, we have conducted a set of numerical simulations using the bubble breakup frequency and daughter bubble PDF models proposed by Martínez-Bazán et al. $^{7,8}$ (Note that as the initial void fraction, $\alpha$, increases,

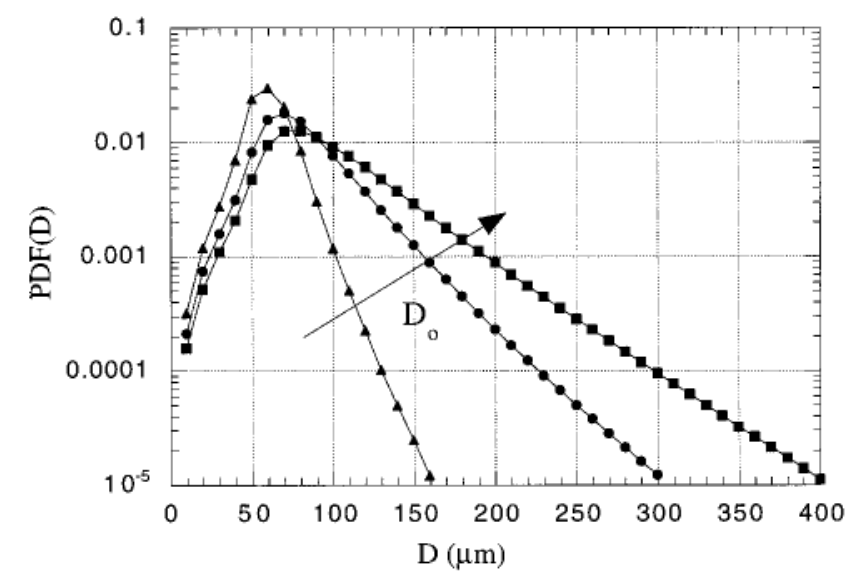

FIG. 6. Computed final $\operatorname{PDF}(D)$ for various sizes of the initial lump of air, $D_{0}$.

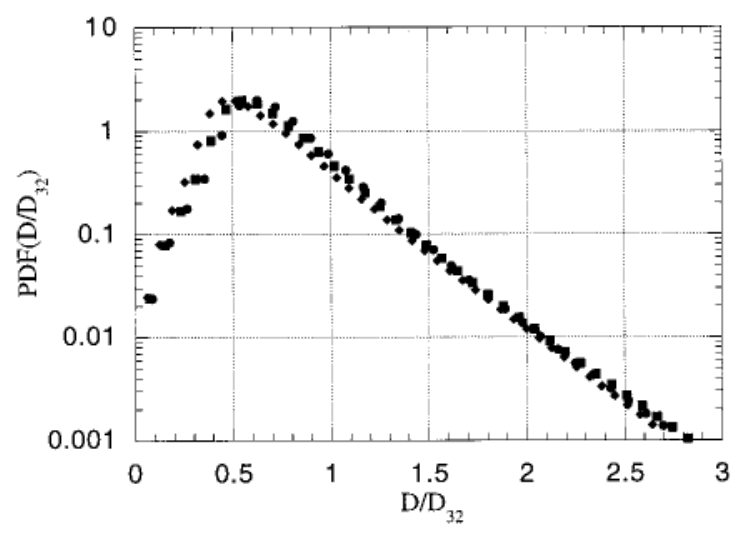

FIG. 7. Nondimensional computed PDF.

the initial lump of air injected into the flow increases and, consequently, $D_{0}$ increases.) In these simulations increasing values of $\alpha$ have been accounted for by increasing the value of the initial bubble size $D_{0}$. The computed bubble size PDFs are shown in Fig. 6 for three different initial bubble diameters, $D_{0}$. Notice the similarity between the PDFs measured experimentally (Fig. 4) and the computed PDF, shown in Fig. 6, obtained with the model. As the size of the initial air bubble, $D_{0}$, increases, the final $\operatorname{PDF}(D)$ becomes wider producing, therefore, larger bubbles. In agreement with the experimental results, when the computed bubble size distributions are normalized with their corresponding Sauter mean diameter, $D_{32}$, all the $\operatorname{PDFs}\left(D / D_{32}\right)$ also collapse onto the same universal curve (see Fig. 7). Thus, one can conclude that the measured dependence of the final PDF on the air void fraction is only due to the effect of the initial bubble size on the bubble breakup frequency and daughter size PDF, and is neither due to coalescence effects nor to a lowering of the local value of the turbulent kinetic energy due to the presence of the bubbles.

\section{ACKNOWLEDGMENTS}

This work has been supported by ONR under Contract No. N00014-96-1-0213. We also acknowledge the support from a Fellowship of the Consejo Asesor the Investigación de la Diputación General de Aragón (Spain) to Carlos Martínez-Bazán. The authors are grateful for the assistance provided to J.L. Montanes by the Spanish Ministry of Education.

${ }^{1}$ A. N. Kolmogorov, "On the breakage of drops in a turbulent flow," Dokl. Akad. Nauk SSSR 66(5), 825 (1949).

${ }^{2}$ J. O. Hinze, "Fundamentals of the hydrodynamics mechanisms of splitting in dispersion process," AIChE. J. 1(3), 289 (1955).

${ }^{3}$ R. D. Cohen, "Shattering of a liquid drop due to impact," Proc. R. Soc. London, Ser. A 435, 483 (1991).

${ }^{4} \mathrm{M}$. S. Longuet-Higgins, "The crushing of air cavities in a liquid," Proc. R. Soc. London, Ser. A 439, 611 (1992).

${ }^{5}$ W. K. Brown and K. H. Wohletz, "Derivation of the Weibull distribution based on physical principles and its connection to the Rosin-Rammler and lognormal distributions," J. Appl. Phys. 78, 2758 (1995).

${ }^{6}$ E. A. Novikov and D. G. Dommermuth, "Distribution of droplets in a turbulent spray," Phys. Rev. E 56(5), 5479 (1997).

${ }^{7}$ C. Martínez-Bazán, J. L. Montañes, and J. C. Lasheras, "On the breakup 
of an air bubble injected into a fully developed turbulent flow. Part I: Breakup frequency," J. Fluid Mech. 401, 157 (1999).

${ }^{8}$ C. Martínez-Bazán, J. L. Montañes, and J. C. Lasheras, "On the breakup of an air bubble injected into a fully developed turbulent flow. Part II: Size PDF of the resulting daughter bubbles," J. Fluid Mech. 401, 183 (1999).
${ }^{9} \mathrm{C}$. Martínez-Bazán, "Splitting and dispersion of bubbles by turbulence," doctoral thesis, University of California, San Diego (1998).

${ }^{10}$ J. C. Lasheras, E. Villermaux, and E. J. Hopfinger, "Break-up and atomization of a round jet by a high speed annular air jet," J. Fluid Mech. 357, 351 (1998) 